\title{
Sustainable Supply Chain Management im globalen Kontext
}

Praxisstand des Lieferantenmanagements in DAX- und MDAX-Unternehmen
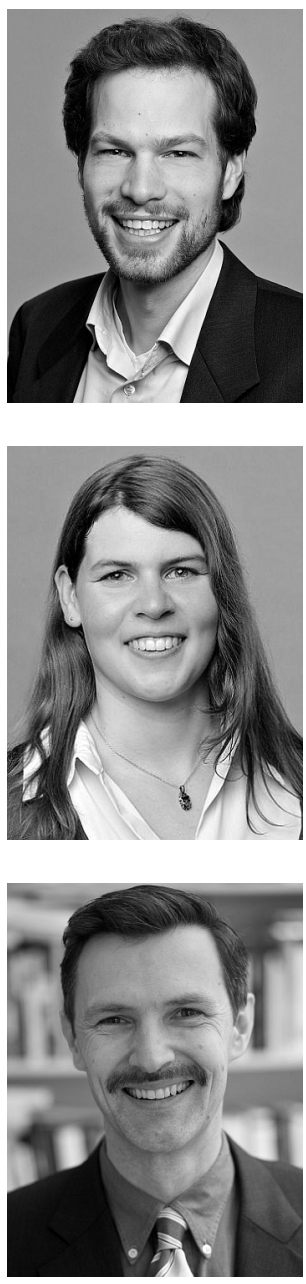

Erik G. Hansen, Dorli Harms und Stefan Schaltegger

Nachhaltigkeit, Sustainability, Lieferantenmanagement, Lieferantenentwicklung, Global Sourcing, Supply Chain

Sustainability, supplier management, supplier development, global sourcing, supply chain management, standards

Das Management globaler Supply Chains mit einer Vielzahl von Lieferanten aus teils sehr unterschiedlichen sozio-ökonomischen Kontexten ist durch hohe Komplexität gekennzeichnet. Die zunehmend geforderte Integration ökologischer und sozialer Aspekte in das konventionelle Lieferkettenmanagement, wie es durch das Sustainable Supply Chain Management (SSCM) repräsentiert wird, steigert diese Komplexität zusätzlich. Damit stellt sich die Frage, wie das SSCM in der Unternehmenspraxis umgesetzt wird. Die vorliegende Studie untersucht die Massnahmen der grössten börsennotierten Unternehmen in Deutschland. Der komparative Vergleich von Umsetzungsmassnahmen des SSCM in DAX- und MDAX-Unternehmen zeigt, dass die Massnahmen bei DAX-Unternehmen häufiger angewendet und formalisierter ausgestaltet werden. Einfach umzusetzende Massnahmen wie schriftliche Anforderungen werden häufiger verwendet als ressourcenaufwendigere Bewertungs- und Audit-Verfahren. Gleichzeitig wird die steigende Bedeutung der Lieferantenentwicklung deutlich.

Global supply chains, often spreading across countries from developed and developing nations, are exposed to diverse socio-economic contexts. The increasing demand for a better control of social and environmental criteria in supply chains, as Sustainable Supply Chain Management (SSCM) argues for, further increases this complexity. This raises the question how SSCM is implemented in corporate practice. The presented survey-based study compares the implementation measures of the largest German stock companies (large caps) with the second largest ones (mid caps). The results show that large caps have progressed further in their implementation and that the processes are more formalized. Measures which are easier to implement, such as written requirements and standards, are more often applied than resource-intensive processes of evaluation and audits. At the same time, the increasing importance of supplier development becomes apparent. 


\section{Einführung}

Aufgrund eines starken Kostendrucks lagern Unternehmen häufig bedeutende Anteile des Wertschöpfungsprozesses an Zulieferer aus Ländern mit komparativ niedrigerem Preisniveau aus und sehen sich somit mit einer höheren Anzahl von Lieferanten und einer grösseren Diversität an Lieferländern konfrontiert (Jahns et al. 2007; Reuter et al. 2010). Das Management globaler Supply Chains (Güter, Information etc.) und die Berücksichtigung von Logistik- und Transportleistungen wird dadurch zunehmend erfolgsrelevant (Arnold/ Eßig 2002, 243; Kotzab et al. 2006; Stölzle/Lukas 2007).

Supply Chain Management (SCM) im globalen Kontext ist jedoch nicht nur aus logistischer Sicht komplex (Goldbach 2001; Stölzle/Heusler 2004; Jahns et al. 2007). Die internationale Verteilung der Zulieferer und die grosse räumliche Distanz beeinflussen die Geschäftsbeziehungen durch sehr stark variierende politische und sozio-ökonomische Rahmenbedingungen, die eine Reihe von ökologischen, sozialen und ökonomischen Nachhaltigkeitsherausforderungen beinhalten (Wittstruck/Teuteberg 2010 a). Dies gilt insbesondere für Zulieferer aus Entwicklungs- und Schwellenländern (Teuscher et al. 2006; Beske et al. 2008). Die globale Verteilung der Lieferanten und starke Zergliederung der Lieferketten führt zu Informations- und Kontrollverlusten des fokalen (d.h. die Wertschöpfungskette kontrollierenden) Unternehmens (Seuring/Müller 2008), zu Reputations- und Produkthaftungsrisiken sowie Optimierungs- und Kontrollschwierigkeiten bei der Berücksichtigung der Nachhaltigkeitsaspekte, die von immer mehr Kunden nachgefragt werden (Goldbach 2001; Bruhn/Meffert 2006; Peters 2010, 28). Reputationsprobleme bei Unternehmen der Bekleidungs-, Lebensmittel- oder Automobilindustrie, die aufgrund von Missständen in Zulieferbetrieben in die Kritik geraten sind (Locke 2003, 40; Zadek 2004; Mamic 2005), gehören zu den zahlreichen bekannten Beispielen, die zeigen, wie bedeutsam ökonomische Nachhaltigkeitsaspekte in Lieferketten sein können.

Vor diesem Hintergrund ist das zunehmende Forschungsinteresse an Sustainable Supply Chain Management (SSCM) - also die Gestaltung globaler Liefer- und Wertschöpfungsketten unter ökologischen, sozialen und ökonomischen Gesichtspunkten - zu erklären (Seuring/Müller 2007; Seuring/Müller 2008; Bai/Sarkis 2010; Gold et al. 2010; Wittstruck/Teuteberg $2010 \mathrm{~b}$ ).

Ein zentraler Aspekt für die Umsetzung des SSCM ist die integrative Berücksichtigung von Nachhaltigkeitsaspekten in das Lieferantenmanagement - d.h. in die Prozesse der Bewertung, Selektion und Entwicklung von Lieferanten (Reuter et al. 2010). Es existieren bisher wenige quantitativ-empirische Studien, die diese Umsetzung von SSCM im Lieferantenmanagement ins Zentrum der Analyse stellen. Ein Teil der Untersuchungen ist rein konzeptionell (Carter/Dresner 2001; Zsidisin/Siferd 2001) oder in Form von Fallstudien aufbereitet (Mamic 2005; Pagell/Wu 2009; Reuter et al. 2010). Bisherige quantitative Studien fokussieren meist auf einzelne Aspekte zur Einführung eines SSCM (Min/Galle 2001; Vachon 2007; Beske et al. 2008) oder konzentrieren sich auf Teilbereiche wie Holt (2004) auf die ökologische Perspektive im SSCM. Andere quantitativ-ökonometrische Studien behandeln hauptsächlich die Verbindung aus SSCM-Massnahmen und finanzieller Performance (Chien/Shih 2007). Aufgrund der dynamischen Veränderungen der letzten Jahre haben viele Untersuchungen an Aktualität verloren (z. B. Beske et al. 2008 mit einer Befragung aus dem Jahr 2003; Holt 2004). Internationale Studien zeigen zudem, dass SSCM bezogen auf die Unternehmensgrösse unterschiedlich ausgestaltet ist (z. B.: Bernstein/ 
Greenwald 2009). In diesem Beitrag wird daher den folgenden Forschungsfragen nachgegangen:

1. Wie wird ein globales SSCM in Bezug auf das Lieferantenmanagement von grossen börsennotierten Unternehmen umgesetzt?

2. Inwiefern unterscheiden sich die Ansätze von sehr grossen und grossen börsennotierten Unternehmen?

Zur Beantwortung der Forschungsfragen dient eine Unternehmensbefragung bei den grössten (DAX) und nächstgrossen (MDAX) Unternehmen an der Deutschen Börse. Dieser Fokus ermöglicht eine hohe Aussagekraft bzgl. des Managements globaler Supply Chains, verfügen einige grosse Unternehmen doch über eine sehr ausgeprägte internationale Wertschöpfungsstruktur (Mamic 2005). Diese Untersuchung nimmt die Perspektive der Agenturtheorie ein, um die Überwindung von Unsicherheit und Informationsasymmetrien zwischen Unternehmen und Lieferanten zu erklären (Williamson 1975; Simpson et al. 2007).

Der Beitrag ist wie folgt gegliedert: Kapitel 2 gibt einen Überblick über Ziele, Treiber und Themen des SSCM. Das dritte Kapitel zeigt auf, wie Nachhaltigkeit in das Lieferantenmanagement integriert werden kann. Kapitel 4 präsentiert die zentralen Ergebnisse der Unternehmensbefragung. Darauf folgt im fünften Kapitel eine Diskussion der Ergebnisse. Kapitel 6 schliesst mit einem kurzen Fazit und Ausblick.

\section{Ziele, Treiber und Themen des SSCM}

Neben den Zielen des konventionellen SCM verfolgt das SSCM weitere Ziele, die von einer Reihe von Treibern unterstützt bzw. von Stakeholdern geäussert werden. Die konkreten Inhalte des SSCM werden dabei einerseits von diesen Stakeholdern benannt. Andererseits entwickeln sich die Ziele des SSCM aus der Kombination des Strebens nach Unternehmenserfolg und diesen Stakeholder-Erwartungen an das SSCM.

\subsection{Ziele des SSCM}

Das konventionelle SCM betrachtet in erster Linie Versorgung, Entsorgung und Recycling über Teile der Lieferkette oder die gesamte Lieferkette (Wildemann 2000, 12; Stölzle) Heusler 2004; Werner 2008, 25 ff.). Dabei werden logistische, informatorische und finanzielle Aspekte optimiert (Gomm 2008; Hofmann/Westerfeld 2010). Bei der Erfüllung dieser Aufgaben wird besonders auf Quantität, Qualität, Kosten, Zeit sowie Liefer- und Lagermodalitäten geachtet. Gut abgestimmte und aktuelle Informationsflüsse sowie die funktionierende Koordination zwischen den Partnern innerhalb der häufig globalen Lieferkette gelten als wesentliche Herausforderungen (Cooper et al. 1997; Stölzle/Heusler 2004).

Von SSCM wird gesprochen, wenn bei der Gestaltung und Optimierung von Lieferketten zusätzlich die Zusammenhänge zwischen ökonomischen, ökologischen und sozialen Aspekten explizit berücksichtigt werden (Seuring/Müller 2008; Bai/Sarkis 2010; Gold et al. 2010; Teuteberg/Wittstruck 2010). Ein besonderes Augenmerk gilt dabei der Integration dieser drei Zieldimensionen (Schaltegger et al. 2007), was auch unter dem Stichwort der „Triple Bottom Line“ diskutiert wird (Elkington 1999; Schaltegger/Burritt 2005). Zu den Zielen des SSCM gehören demnach die Reduktion sozialer und ökologischer Risiken in der Lieferkette und die Realisierung von Chancen, z. B. durch kooperativ entwickelte Produkt- und Prozessinnovationen. Vorrangiges Ziel ist eine über die Unternehmensgrenzen hinausgehende, unternehmerische Entwicklung des Wertschöpfungsnetzwerks, das zu 
einer nachhaltigen Entwicklung beiträgt und sich positiv auf den Unternehmenserfolg auswirkt (Pagell/Wu 2009).

\subsection{Treiber des SSCM}

Die vermehrte Befassung mit SSCM wird durch unternehmensexterne und -interne Treiber angeregt. Als wichtige externe Treiber für das SSCM gelten auf der Regulierungsebene die Gesetzgebung (Hall 2000; Carter/Dresner 2001; Min/Galle 2001; Walker et al. 2008), auf Marktebene die Kunden und Wettbewerber (Lamming/Hampson 1996; Klassen/Vachon 2003; Zhu/Sarkis 2006) und auf gesellschaftlicher Ebene die NGOs und die Öffentlichkeit (Sharma/Vredenburg 1998; Wycherly 1999; Hall 2000; Preuss 2001; Koplin et al. 2007). Entweder auf Basis dieser externen Treiber oder aufgrund eigener Wertvorstellungen treten auch intern verschiedene Akteure als Treiber in Erscheinung, insbesondere die Geschäftsführung und die Nachhaltigkeitsabteilung (Wycherly 1999; New et al. 2000).

Die Interessen der identifizierten Treiber sind für das fokale Unternehmen mit einigen Risiken und Chancen verbunden. Ein Risiko ist die Produkthaftung, die teilweise die Verantwortung für Zulieferer einschliesst (Loew 2006; Winkler et al. 2007, 39). Durch die Entwicklung des Internets und der Social Media haben Reputationsrisiken stark zugenommen, ermöglichen sie doch eine schnelle Informationsverbreitung über in beliebigen Wertschöpfungsgliedern vorhandene, soziale oder ökologische Missstände (Koplin et al. 2007). Durch das gestiegene Bewusstsein für Nachhaltigkeitsthemen von Konsumenten ist dies besonders kritisch (Bruhn/Meffert 2006; BMU 2008). Diesem marktseitigen Risiko steht die Chance gegenüber, hinsichtlich einer ansteigenden Nachfrage von Produkten mit hohen ökologischen und sozialen Standards, Differenzierung zu erreichen (Reuter et al. 2010).

\subsection{Themen und Standards des SSCM}

Unter dem Begriff der Nachhaltigkeit sehen sich Unternehmen einer Vielzahl verschiedener ökologischer und sozialer Themen wie Kinder- und Zwangsarbeit, Materialverbrauch oder Erhalt der Artenvielfalt gegenüber, die entlang der Wertschöpfungskette von Bedeutung sein können (Mamic 2005). Eine sehr umfangreiche, weiter ausdifferenzierte Liste von Nachhaltigkeitsthemen generell für die Unternehmensführung enthalten die internationalen Richtlinien zur Nachhaltigkeitsberichterstattung der Global Reporting Initiative (GRI 2006). Des Weiteren sind für das SSCM Standards von Bedeutung, die Teilaspekte dieser ökologischen und sozialen Themen explizit für die Lieferkette bzw. das SSCM einbeziehen (Beske et al. 2008; Seuring/Müller 2008). Zunächst kann unterschieden werden zwischen sozial- bzw. gesellschaftsorientierten Standards (z. B. Kernarbeitsnormen der Internationale Labour Organization (ILO); Global Compact) sowie ökologieorientierten (z. B. EcoManagement and Audit Scheme (EMAS); DIN ISO 14001) und wirtschaftlich ausgerichteten Standards (z. B. im Sinne von Qualität: DIN ISO 9000; OECD Leitlinien). Dabei zeigt sich, dass diese Standards z. T. mehr als eine Nachhaltigkeitsdimension ansprechen (Koplin 2006, 223).

Parallel dazu nutzen Unternehmen firmen- oder brancheneigene (Verhaltens-)Kodizes, sogenannte Codes of Conduct, um die Umwelt- und Sozialwirkungen der Lieferanten zu steuern (Kolk et al. 1999, 152; Handfield et al. 2002). Codes of Conduct sind schriftlich niedergelegte Richtlinien, die als Grundlage für das Verhalten von Unternehmen gegen- 
über ihren Zulieferern, der Belegschaft oder anderen Akteuren, mit denen ein Unternehmen Geschäftsbeziehungen pflegt, dienen sollen (Mamic 2005). Sie sind i.d.R. spezifischer als internationale Standards auf die jeweiligen Charakteristika der Branche und der betroffenen Länder und Kulturen ausgerichtet und greifen als Branchen-Kodizes häufig einer drohenden staatlichen Regulierung vor (Kolk et al. 1999, 152).

Codes, Normen und Standards dienen im Lieferantenmanagement häufig auch als Bewertungs- und Auswahlkriterium, auf das im Folgenden näher eingegangen wird.

\section{SSCM und das Lieferantenmanagement}

Ein globales SSCM ist charakterisiert durch interorganisationale Zusammenarbeit über nationale und kulturelle Grenzen hinweg und ist damit Risiken ausgesetzt (Teuteberg/ Wittstruck 2010). Unternehmen haben üblicherweise keine genaue Einsicht in die ökologischen und sozialen Bedingungen vor Ort bei den Lieferanten (De Nardo et al. 2010). Aus agenturtheoretischer Sicht besteht eine grosse Unsicherheit aufgrund dieser hohen Informationsasymmetrie zwischen fokalen Unternehmen (Prinzipal) und Lieferanten (Agenten). Diese Asymmetrie nimmt mit jeder vorgelagerten Stufe (Vorlieferanten) weiter zu und kann durch Lieferanten opportunistisch ausgenutzt werden (Coase 1937; Williamson 1975; Picot et al. 2003, 59). Diese Überlegungen sind zu beachten, wenn Nachhaltigkeitsaspekte in das Lieferantenmanagement und dessen Handlungsfelder integriert werden (Carter/Jennings 2004; Koplin et al. 2007; Simpson et al. 2007; Müller et al. 2009; Reuter et al. 2010). Die Handlungsfelder können unterschieden werden in: Bewertung, Selektion und Entwicklung (Reuter et al. 2010).

\subsection{Lieferantenbewertung und -selektion}

Im Zentrum agenturtheoretischer Überlegungen bei der Lieferantenbewertung und -selektion steht das Problem der falschen Lieferantenauswahl vor Vertragsabschluss (Adverse Selection) sowie das Problem, das Verhalten des Lieferanten nach Vertragsabschluss nicht beurteilen zu können (Moral Hazard). Die systematische Bewertung und Selektion sowie die Ausgestaltung expliziter Verträge sind daher zentrale Bestandteile des Lieferantenmanagements (Reuter et al. 2010).

Als konkrete Instrumente zur Lieferantenbewertung dient häufig eine Selbsteinschätzung der Lieferanten mittels Fragebögen (Mamic 2005). Weiterhin sind Zertifizierungen und Audits vor Ort, jeweils durch Vertreter des fokalen Unternehmens selbst oder aber mit Hilfe externer Dienstleister, möglich. Insbesondere die Auditierung und Zertifizierung von Lieferanten durch branchenübergreifende Organisationen oder externe Dienstleister hat an Bedeutung gewonnen (Vachon 2007). Die bereits erwähnten Codes of Conduct, Normen und Standards können als Basis für die Bewertung und Zertifizierung dienen (Walton et al. 1998; Holt 2004). Die verschiedenen Anforderung und Bewertungsmechanismen können auch innerhalb von Lieferantenverträgen eingebunden werden, mit deren Verhandlung und Durchsetzung allerdings Transaktionskosten entstehen (Mamic 2005; Simpson et al. 2007).

Zusätzlich zu Labels und Zertifikaten, welche die Einhaltung von Umwelt- und Sozialstandards im Voraus bescheinigen (Signalling), kann auch die Lieferantenbewertung als Entscheidungskriterium in die Lieferantenselektion einfliessen. Darüber hinaus wird die Bewertung als Bestandteil eines kontinuierlichen Monitorings genutzt (Walton et al. 1998; 
Holt 2004). Mit dem Monitoring sind üblicherweise Anreize oder Sanktionen verbunden. Bei negativen Evaluierungsergebnissen können Lieferanten auch ausgelistet werden (Darnall/Carmin 2005; Delmas/Montiel 2009). Eine Beendigung der Lieferantenbeziehung bedeutet allerdings auch die Suche und Auswahl eines neuen Lieferanten, was mit zusätzlichen Transaktionskosten verbunden ist und nicht garantiert, dass der neue Lieferant in der Umsetzungspraxis dann tatsächlich im Sinne der Erfüllung von umwelt- und sozialorientierten Anforderungen besser arbeitet. Eine Alternative zur Auslistung eines Lieferanten kann darin bestehen, Lieferanten mit Schulungsmassnahmen zu entwickeln.

\subsection{Lieferantenentwicklung}

Wenn auch im geringeren Masse als durch Bewertung und Monitoring (Holt 2004), spielt das Instrument der Lieferantenentwicklung eine zunehmende Bedeutung für das SSCM (Schaltegger et al. 2007; Seuring/Müller 2008; Vachon et al. 2009; Reuter et al. 2010). Sie dient der Qualifizierung der Lieferanten zu Nachhaltigkeitsthemen und ermöglicht so - im Gegensatz zum Lieferantenwechsel - längerfristige Geschäftsbeziehungen. Die Lieferantenentwicklung dient zudem der Reduzierung von Informationsasymmetrien (und damit der Verhinderung eines Moral Hazards), denn im Rahmen von Entwicklungsmassnahmen kann ein (im Unterschied zum Monitoring) noch detaillierteres Bild der Lieferanten entstehen.

Mögliche Entwicklungsinstrumente sind beispielsweise Gespräche mit den Lieferanten, um das Bewusstsein für die Bedeutung der Nachhaltigkeitsaspekte zu schärfen oder Verbesserungsmassnahmen zu vereinbaren (Mamic 2005; Delmas/Montiel 2009). Weiterhin können konkrete Umsetzungsprojekte (z. B. um die $\mathrm{CO}_{2}$-Emissionen zu verringern) gemeinsam durchgeführt oder in Schulungen und Trainingsmassnahmen investiert werden (Mamic 2005; Reuter et al. 2010). Durch gemeinsame Projekte entstehen zwar z. B. Koordinationskosten, Informationskosten und -unsicherheiten werden jedoch reduziert.

\section{Empirische Untersuchung der Managementpraxis von DAX- und MDAX-Unternehmen}

\subsection{Methodisches Vorgehen}

Die vorliegende empirische Untersuchung basiert auf einer umfassenden Studie zum Praxisstand des SSCM in DAX- und MDAX-Unternehmen. Der DAX umfasst die nach Marktkapitalisierung und Börsenumsatz grössten und der MDAX die nächstgrossen Unternehmen (Deutsche Börse 2007). Auch andere Studien, die internationale Wertschöpfungsketten analysieren, unterscheiden zwischen Unternehmen hinsichtlich der Marktkapitalisierung (Bernstein/Greenwald 2009). Wie Tabelle 1 zeigt, spiegeln sich die Grössenunterschiede der beiden hier untersuchten Unternehmensgruppen im Durchschnitt auch bezüglich Jahresumsatz und Mitarbeiterzahl wider, obgleich Werte für einzelne Unternehmen leicht abweichen. Die Analyse basiert auf einer schriftlich-postalischen Erhebung mit standardisiertem Fragebogen (mit geschlossenen und offenen Fragen), die von September 2008 bis Januar 2009 durchgeführt wurde (eine Übersicht von Fragen befindet sich in Tabelle 4 im Anhang). Insgesamt nahmen 32 Unternehmen (15 DAX, 17 MDAX) an der Befragung teil (vgl. Tabelle 1 für die Branchenverteilung nach der Deutschen Börse). Für die empirische Untersuchung wurden die an der Börse notierten 30 DAX- und 50 MDAX-Unternehmen zunächst telefonisch kontaktiert, wobei acht der MDAX-Unternehmen eine Teilnahme an der darauf folgenden schriftlichen Befragung grundsätzlich ablehnten. 15 
der insgesamt 30 DAX- und 17 der verbleibenden 42 MDAX-Unternehmen haben einen ausgefüllten Fragebogen zurückgesendet. Dies entspricht einer Rücklaufquote von 50\% bei DAX und $40 \%$ bei MDAX. Die kontaktierten Unternehmensvertreter waren in den Bereichen Einkauf/Supply Chain Management/Logistik (57\%), Nachhaltigkeit/Quality, Health, Safety, Environment $(25 \%)$ und anderen Bereichen $(9 \%)$ tätig. Einige der Personen $(9 \%)$ beantworteten die Frage zur organisatorischen Zuordnung nicht.

\begin{tabular}{lll}
\hline Charakteristika & DAX & MDAX \\
\hline Anzahl Unternehmen & 15 & 17 \\
$\varnothing$ Mitarbeiterzahl & 165.258 & 23.894 \\
$\varnothing$ Jahresumsatz in Mio. Euro (Anzahl Unternehmen) ${ }^{1}$ & $52.298(11)$ & $7.648(14)$
\end{tabular}

Branchenzuordnung

(nach Sektoren der Deutschen Börse)

- Banken/Versicherung

- Chemie/Pharma

- Handel und Transport/Logistik

- Industrie/Konsumgüter

- Andere

$4(27 \%)$

$3(20 \%)$

$1(6 \%)$

$3(20 \%)$

$4(27 \%)$

$15(100 \%)$
$3(18 \%)$

$3(18 \%)$

$2(11 \%)$

$6(35 \%)$

$3(18 \%)$

$17(100 \%)$

- Gesamt

Tabelle 1: Übersicht des Samples (basierend auf Daten der Geschäftsberichte aus 2007/2008)

In der vorliegenden Empirie werden zunächst die Charakteristika der Wertschöpfungsketten der zwei Unternehmensgruppen, die wichtigsten Treiber für das SSCM und die Relevanz unterschiedlicher sozialer und ökologischer Themen dargestellt. Dann werden die Massnahmen zur Integration von Nachhaltigkeit in das Lieferantenmanagement untersucht.

\subsection{Charakteristika der Supply Chain, Treiber und Nachhaltigkeitsthemen}

\subsubsection{Komplexität der internationalen Supply Chains}

Im Vergleich zu MDAX-Unternehmen haben die grösseren DAX-Unternehmen eine höhere Lieferantenanzahl (mehr als 5.000 Lieferanten: 73\% DAX; 35\% MDAX) und lassen aus einer grossen Anzahl von Ländern liefern (mehr als 50 Beschaffungsländer: 60\%

111 der 15 DAX-Unternehmen und 14 der 17 MDAX-Unternehmen weisen in ihren Geschäftsberichten einen Jahresumsatz aus; die verbleibenden 4 DAX-Unternehmen und 3 MDAX-Unternehmen sind Banken bzw. Versicherungen, die in ihren Geschäftsberichten die Bilanzsumme bzw. Bruttobeiträge angeben. Da die beiden letztgenannten Grössen nicht mit dem Jahresumsatz vergleichbar sind, werden sie hier nicht zur Durchschnittsberechnung herangezogen. Hinsichtlich der Grössenordnung ist jedoch ähnlich zum Jahresumsatz festzustellen, dass Bilanzsummen bzw. Bruttobeiträge bei DAX-Unternehmen im Vergleich zu MDAX-Unternehmen im Durchschnitt deutlich höher liegen (5-bis 31-fach höher). 
DAX; 35\% MDAX). DAX-Unternehmen beziehen häufiger als MDAX-Unternehmen aus Schwellen- und Entwicklungsländern (während aus ähnlich vielen Ländern Asiens geliefert wird, beziehen DAX-Unternehmen aus 9-31\% mehr Ländern aus Afrika und Lateinamerika Produkte bzw. Dienstleistungen). Des Weiteren ist bei beiden Unternehmensgruppen eine deutliche Internationalisierungstendenz der Produktions- bzw. Dienstleistungsstandorte erkennbar (73\% der DAX- und 76\% der MDAX-Unternehmen geben dies an).

Wird nach Gründen gefragt, die für die Komplexität in Lieferantenbeziehungen ausschlaggebend sind (vgl. Abbildung 1), zeigt sich, dass die DAX-Unternehmen in der Tendenz fast alle abgefragten Gründe als ausschlaggebender bewerten als MDAX-Unternehmen (mit Ausnahme der „Geringen Währungsstabilität“). Insgesamt beurteilen die befragten Unternehmen ihre internationalen Lieferantenbeziehungen i.d.R. als komplex bis sehr komplex (80\% DAX; 82\% MDAX). Die seitens der Unternehmen genannten Gründe spiegeln dabei die besondere Rolle der Internationalität in der Komplexität der Lieferkette wider. Andere empirische Studien bestätigen die durch die Unternehmensgrösse induzierte, zunehmende Internationalität, insbesondere hinsichtlich der Entwicklungs- und Schwellenländer (Reuter et al. 2010).

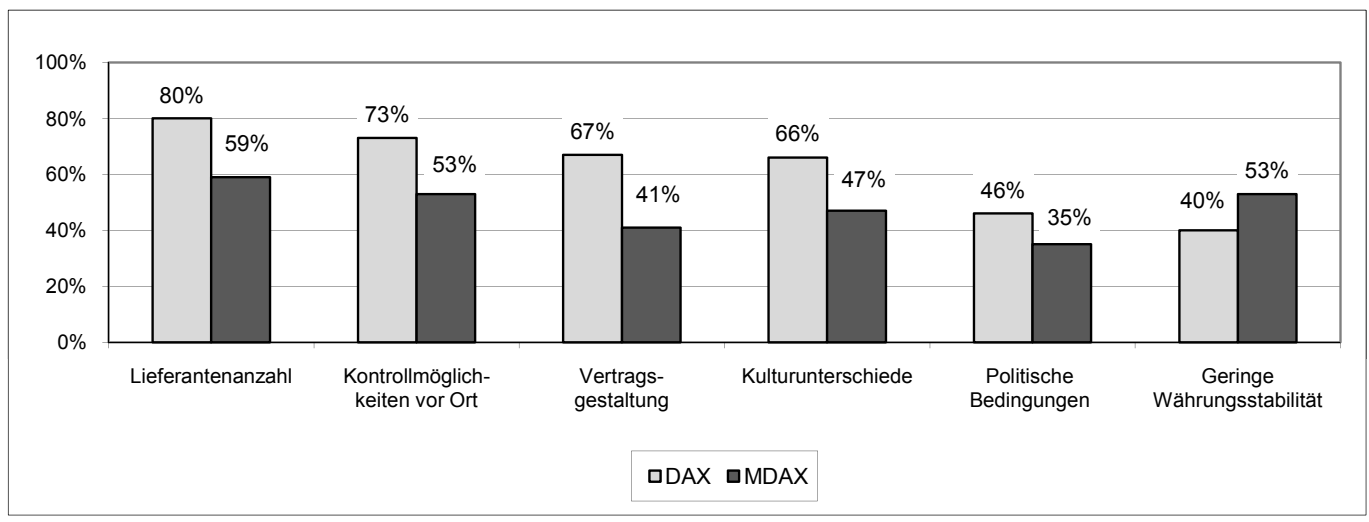

Abbildung 1: Gründe für Komplexität in Lieferantenbeziehungen (Antworten „ausschlaggebend“/,sehr ausschlaggebend“ zusammengefasst)

Die befragten Unternehmen ordnen in der Supply Chain vielen Nachhaltigkeitsthemen eine grosse Bedeutung zu (jeweils Minimalwert der Nennungen von DAX oder MDAX): Abfallreduktion $(\geq 88 \%)$, Gesundheitsschutz $(\geq 87 \%)$, Kinder- und Zwangsarbeit $(\geq 82 \%)$, Menschenrechte $(\geq 82 \%)$, Reduktion von Treibhausgas-Emissionen $(\geq 82 \%)$, Verminderung von negativen Umwelteinflüssen $(\geq 82 \%)$, Gleichberechtigung $(\geq 80 \%)$, Material- und Ressourcenverbrauch ( $\geq 71 \%$ ), Vereinigungsfreiheit $(\geq 71 \%)$ und Arbeitsplatzsicherheit $(\geq 65 \%)$. Trotz einer insgesamt hohen Relevanz in DAX- und MDAX-Unternehmen zeigt sich im Detail, dass die DAX-Gruppe mit wenigen Ausnahmen (Gesundheitsschutz; Kinder- und Zwangsarbeit) die Themen höher gewichtet als die MDAX-Gruppe. Darüber hinaus ist feststellbar, dass die Bedeutung erneuerbarer Energien in der DAXGruppe $(80 \%)$ sehr viel grösser ist als bei der MDAX-Gruppe (47\%). Ausserdem wird dem Thema der „Artenvielfalt“ (als Teilaspekt der Biodiversität) trotz internationaler und nationaler Aktionsprogramme (z. B. nationale Biodiversitätsstrategie der deutschen Bun- 
desregierung (BMU 2007) für die Supply Chain die geringste Bedeutung beigemessen (27\% DAX; 24\% MDAX). Diese geringe Bedeutung zeigt sich auch in neueren empirischen Untersuchungen zum unternehmerischen Nachhaltigkeitsmanagement (Schaltegger et al. 2010).

Die als am bedeutendsten bewerteten Themen des SSCM, Abfallreduktion, Gesundheitsschutz, Kinder- und Zwangsarbeit, Menschenrechte, Reduktion von Treibhausgas-Emissionen und Verminderung von negativen Umwelteinflüssen, sind häufig nahe an den Produktionsprozessen verortet. Weniger Gewicht werden tendenziell Themen wie Artenvielfalt oder Arbeitsplatzsicherheit beigemessen, die nur indirekt oder mittelbar mit den Produktions- und Logistikprozessen in Verbindung gebracht werden können. Dabei sind nur wenige Unterschiede in der Gewichtung ökologischer gegenüber sozialen Themen festzustellen.

Die als relevant angesehen Nachhaltigkeitsthemen verdeutlichen, dass das SSCM im Vergleich zum konventionellen SCM insgesamt mit einer höheren Komplexität umgehen muss (Goldbach 2003; Seuring et al. 2004; Kumar/Malegeant 2006; Gießmann/Lasch 2010). Dennoch befassen sich immer mehr Unternehmen mit SSCM. Hierzu tragen eine Reihe von Treibern bei.

\subsubsection{Treiber}

Das SSCM wird durch eine Vielzahl von Treibern ausserhalb und innerhalb der Unternehmen befördert. Die wichtigsten zukünftigen externen Treiber der DAX-Unternehmen, beinahe gleich häufig genannt, sind Kunden, Gesetzgebung, Aktionäre (jeweils 60\%) und Medien (53\%). Die hohe Bedeutung dieser Stakeholder zeigt sich auch in vorausgegangenen Studien (vgl. u.a. Carter/Dresner 2001; Walker et al. 2008). Diese vier Treiber sind auch im MDAX am wichtigsten, aber mit grösseren relativen Unterschieden in der Häufigkeit der Nennungen (Kunden 82\%; Gesetzgebung 76\%; Aktionäre 53\%; Medien 47\%). Im Vergleich zeigt sich, dass bei MDAX-Unternehmen Kunden und Gesetzgebung hinsichtlich ihres Prozentanteils häufiger genannt werden als bei DAX-Unternehmen; für Medien und Aktionäre ergibt sich ein umgekehrtes Bild.

Aufgrund des vorliegenden Untersuchungsfokus auf die Umsetzung von SSCM, werden im Folgenden insbesondere die unternehmensinternen Treiber für das SSCM diskutiert (Abbildung 2). 


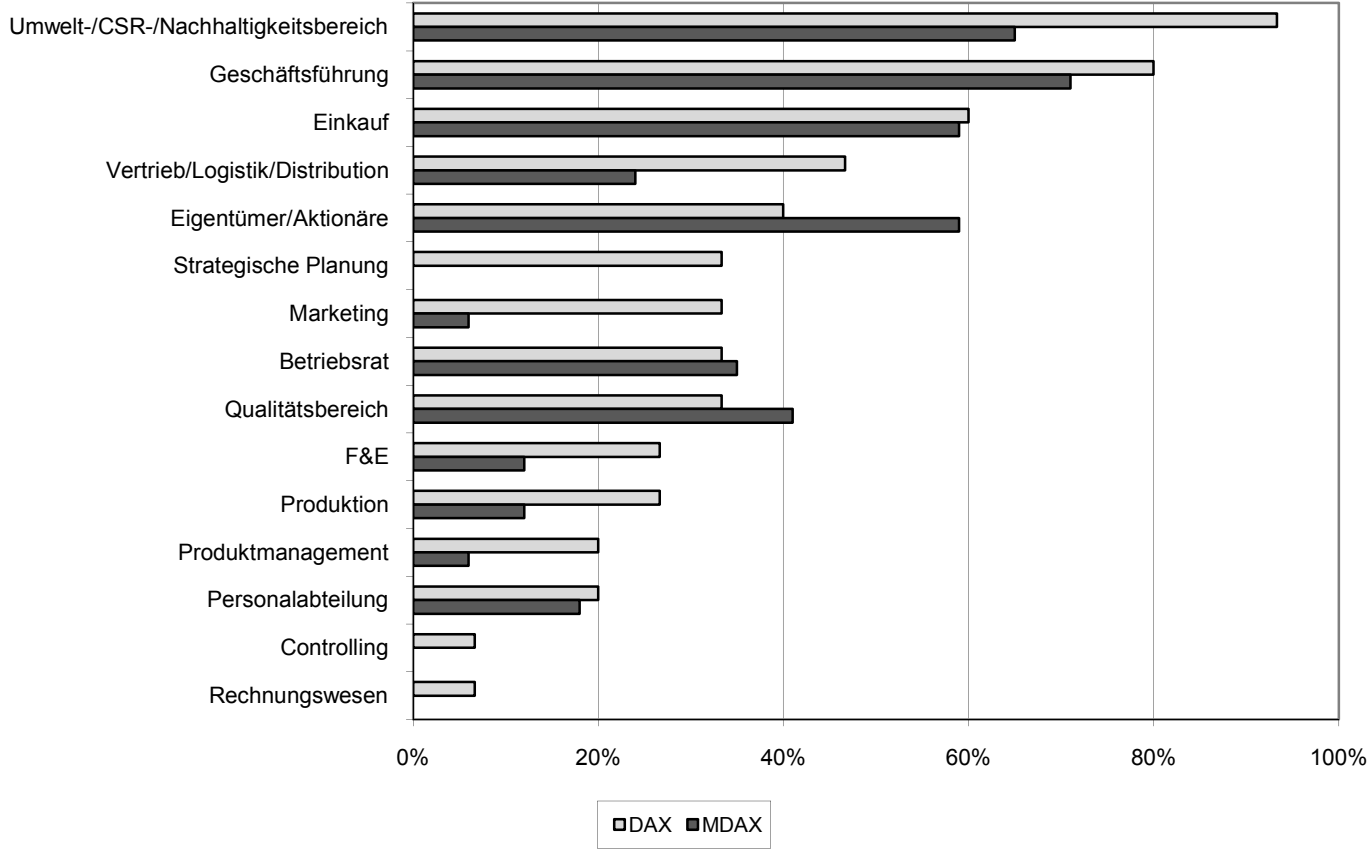

Abbildung 2: Interne Treiber für das SSCM

Wie in Abbildung 2 dargestellt, werden von mehr als der Hälfte der DAX- und MDAXUnternehmen sowohl der Nachhaltigkeitsbereich (bzw. Umweltbereich) als auch die Geschäftsführung und der Einkauf am häufigsten als treibende Kräfte für das SSCM gesehen (mind. 59\%). Die bedeutende Rolle der Geschäftsleitung für die Umsetzung von SSCM wird vielfach genannt (vgl. u.a. Pagell/Wu 2009). Allerdings zeigen Carter et al. (1998) auch, dass ökologische Beschaffung eher durch das mittlere Management unterstützt wird. Auffällig ist, dass marktseitige Funktionsbereiche (Vertrieb/Logistik/Distribution, Produktmanagement und Marketing) sehr viel häufiger von den DAX- als von den MDAX-Unternehmen als interne Treiber benannt werden.

Die Ergebnisse lassen den Schluss zu, dass bei den kleineren MDAX-Unternehmen die zentrale Verantwortung für Nachhaltigkeitsthemen und das SSCM meist nicht in einer spezifischen Nachhaltigkeitsabteilung liegt, sondern im Qualitäts- oder Personalmanagement als Zusatzaufgabe verortet wird. Weiterhin zeigt sich, dass die sonstigen Funktionen - insbesondere die marktlichen (z. B. Vertrieb, Marketing) - beim MDAX viel seltener als Treiber auftreten. Bei ihnen scheint noch keine systematische Integration in die weiteren Funktionsbereiche stattgefunden zu haben. Eher wird Nachhaltigkeit als „parallele Organisation " in den oben erwähnten, hauptverantwortlichen Stellen betrieben (Schaltegger) Wagner 2006; Hansen 2010, 143). 


\subsection{Massnahmen im Lieferantenmanagement}

\subsubsection{Anforderungen, Bewertung und Auswahl}

Für die Umsetzung des SSCM im Lieferantenmanagement formulieren Unternehmen z. B. in Form von Standards explizite Anforderungen an ihre Lieferanten (vgl. Abschnitt 2.3), um beispielsweise der Gefahr von Adverse Selection vorzubeugen. Tabelle 2 zeigt, dass Anforderungen von DAX-Unternehmen häufiger als von MDAX-Unternehmen schriftlich festgehalten werden. Weiterhin lässt sich für beide Gruppen feststellen, dass eine explizit schriftliche Fixierung eher für ökologische Anforderungen als für soziale stattfindet. Ein bedeutender Unterschied besteht auch in der Rolle der schriftlichen Fixierung bei eigener Bewertung/Auditierung im Kontrast zu derjenigen durch externe Dienstleister.

\begin{tabular}{|l|ll|lc|}
\hline \multirow{2}{*}{ Anforderung } & \multicolumn{2}{|c|}{ Ökologisch } & \multicolumn{2}{c|}{ Sozial } \\
\cline { 2 - 5 } & DAX & MDAX & DAX & MDAX \\
\hline Mindeststandards & $100 \%$ & $71 \%$ & $93 \%$ & $59 \%$ \\
Lieferverträge, AGBs & $87 \%$ & $71 \%$ & $80 \%$ & $65 \%$ \\
Code of Conduct & $87 \%$ & $47 \%$ & $80 \%$ & $41 \%$ \\
Audits durch eigenes Personal & $60 \%$ & $71 \%$ & $60 \%$ & $53 \%$ \\
Eigene Lieferantenbewertung & $67 \%$ & $65 \%$ & $60 \%$ & $47 \%$ \\
Audits durch externe Dienstleister & $27 \%$ & $29 \%$ & $33 \%$ & $24 \%$ \\
Externe Lieferantenbewertung & $33 \%$ & $18 \%$ & $27 \%$ & $24 \%$ \\
\hline
\end{tabular}

Tabelle 2: Anteil der Unternehmen, die ökologische/soziale Anforderungen in Verträgen/ Vereinbarungen mit ihren Lieferanten explizit schriftlich festhalten

Die erkennbare stärkere Gewichtung ökologischer Anforderungen (im Vergleich zu sozialen) steht dem Ergebnis gegenüber, dass in DAX- und MDAX-Unternehmen Umwelt- und Sozialthemen etwa gleich relevant sind (vgl. Abschnitt 4.1.1). Diese Abweichung kann darauf zurückgeführt werden, dass Themen zwar ähnlich relevant sind, die ökologischen Aspekte jedoch besser zu quantifizieren und kontrollieren sind und somit besser als Anforderung dienen können (Richards/Gladwin 1999; Schaltegger et al. 2007). Dies erweitert die Erkenntnis aus der Literatur, in der allgemein eine höhere Bedeutung ökologischer Themen beobachtet werden kann (Seuring/Müller 2008).

Unter der Annahme, dass grundsätzlich die gleichen ökologischen/sozialen Massstäbe bei internen oder externen Bewertungen angesetzt werden, zeigen sich dennoch Unterschiede bei der entsprechenden Durchführung. So ist zu erkennen, dass eigene Lieferantenbewertung und Audits im Unterschied zur Durchführung durch externe Dienstleister etwa doppelt so häufig angewendet werden, und dies, obwohl eine zunehmende Anzahl externer Dienstleister am Markt vorhanden ist (Mamic 2005). Die interne Durchführung scheint mehr Freiheitsgrade zuzulassen und den eigenen Kompetenzaufbau zu fördern. Insbesondere unter externem Legitimitätsdruck kann es jedoch erforderlich sein, die zunächst interne Durchführung auf (unabhängigere) externe Dienstleister zu übertragen 
(Zadek 2004). Einschränkend ist jedoch zu erwähnen, dass externe Prüfer nicht immer unabhängig sind (Mamic 2005; Müller et al. 2009).

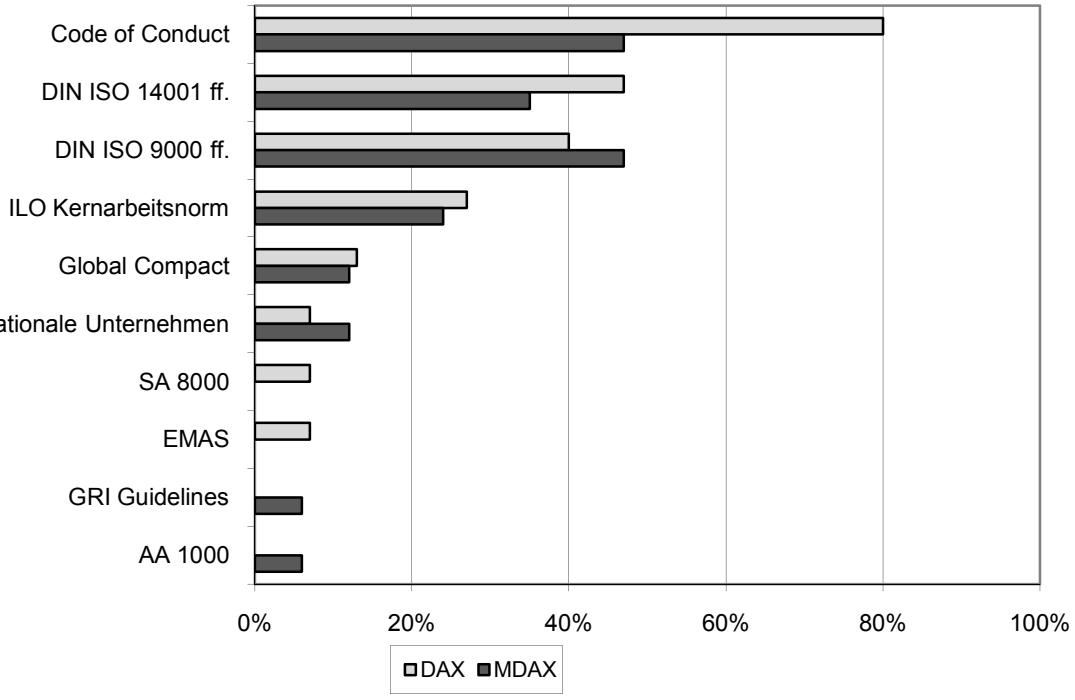

Abbildung 3: Anteil der Unternehmen, die von „vielen bis allen“ Lieferanten die Einhaltung von Standards verlangen

\subsubsection{Einhaltung von Standards}

Grundsätzlich kann jedes Unternehmen von seinen Lieferanten im Sinne von Signalling Nachweise über die Einhaltung von Qualitäts-, Umwelt- und Sozialstandards einfordern. Die DAX-Unternehmen geben an, bei ihren Lieferanten am häufigsten über Codes of Conduct oder die bekannten Standards und Normen (z. B. ISO 9000 und 14001) Nachweise zu verlangen (Abbildung 3). Ähnliches gilt für die MDAX-Unternehmen, wobei Codes of Conduct eine sehr viel geringere Rolle spielen und zu Umweltmanagementstandards etwas weniger häufig ein Nachweis verlangt wird (35\%).

Einerseits bestätigen die empirischen Ergebnisse, dass einige Standards wie SA 8000 und AA 1000 wegen ihrer Branchenspezifität nur wenig eingesetzt werden, andererseits widersprechen sie der üblicherweise weiteren Verbreitung des EMAS-Standards (Koplin et al. 2007; Beske et al. 2008). Die globalen Supply Chains der hier untersuchten Unternehmen sprechen dafür, dass in diesem globalen Umfeld die internationalere ISO 14001-Norm dem EMAS-Standard vorgezogen wird.

Wird darüber hinaus nach der Anzahl der Vorlieferanten gefragt, von denen ein Nachweis über die Einhaltung von Nachhaltigkeitsstandards verlangt wird, zeigt sich, dass dies im Gegensatz zu den direkten Lieferanten kaum eine Rolle spielt (maximal 13\% der DAXbzw. $12 \%$ der MDAX-Unternehmen geben an, entsprechende Nachweise zu verlangen). Hier liegt die Erklärung nahe, dass dies an der noch grösseren Anzahl von Vorlieferanten und deren schwierige Erreichbarkeit auf vorgelagerten Stufen liegt. Anstatt direkte Massnahmen zum Abbau der stark ansteigenden Informationsasymmetrien bzgl. der Vorlieferanten zu ergreifen, können „Trickle Down“- oder „Green Multiplier“-Effekte (Preuss 2001; Holt 2004) erwägt werden, bei denen Lieferanten ihrerseits Massnahmen bei den 
Vorlieferanten durchsetzen. „Trickle down“ bedeutet hier, dass Wirkungen der vom fokalen Unternehmen getroffenen Massnahmen von den direkten Lieferanten zu weiteren Vorstufen in der Lieferkette „durchsickern“.

\subsubsection{Lieferantenentwicklung}

Unternehmen verfügen über ein breites Sanktions- und Massnahmenspektrum, um Sozialund Umweltleistungen bei ihren Lieferanten nach Vertragsabschluss zu verbessern (Tabelle 3). DAX-Unternehmen legen dabei bereits in den Liefervereinbarungen Sanktionsmöglichkeiten deutlich häufiger schriftlich und mündlich fest $(74 \%)$ als MDAX-Unternehmen (36\%), wie zudem aus Abbildung 4 ersichtlich ist. Wird nach der Häufigkeit des Ergreifens von entsprechenden Standard-Massnahmen (Massnahmen die „immer“ durchgeführt werden) bei Lieferanten gefragt, zeigt sich, dass bei DAX-Unternehmen die folgenden Massnahmen am häufigsten genutzt werden (mit sinkender Bedeutung): Verwarnung, Potenzial-Gespräche, Beendigung der Lieferantenbeziehung und gleichermassen eigene Kontrollen sowie Schulungen. Die MDAX-Unternehmen scheinen in ihrem Standardvorgehen wesentlich weniger kooperativ zu sein: Hier wird seltener verwarnt, es werden weniger Gespräche geführt oder Schulungen durchgeführt, dafür aber öfter eigene Kontrollen vor Ort vorgenommen und die Lieferantenbeziehung beendet. Bei den Massnahmen, die fallspezifisch (d.h. „manchmal“) durchgeführt werden, relativiert sich zumeist die schwächere Ausprägung der MDAX-Unternehmen, mit Ausnahme der Schulungsmassnahmen.

Dass DAX-Unternehmen wesentlich stärker in kooperative Lieferantenentwicklung investieren, ist aus Sicht der Ressourcensicherung verständlich, kann aber auch durch das höhere Engagement in Entwicklungs- und Schwellenländern erklärt werden, da in diesen Ländern das Risiko besonders hoch und der Bedarf nach Know-how-Aufbau ausgeprägt ist (Teuscher et al. 2006; Beske et al. 2008).

\begin{tabular}{|c|c|c|c|c|c|c|}
\hline \multirow[b]{2}{*}{ Massnahmen } & \multicolumn{2}{|c|}{ Immer } & \multicolumn{2}{|c|}{ Manchmal } & \multicolumn{2}{|c|}{$\begin{array}{l}\text { Nie / k.A. / weiss } \\
\text { nicht }\end{array}$} \\
\hline & DAX & MDAX & DAX & MDAX & DAX & MDAX \\
\hline Eigene Kontrolle vor Ort & $13 \%$ & $24 \%$ & $67 \%$ & $71 \%$ & $20 \%$ & $5 \%$ \\
\hline $\begin{array}{l}\text { Gespräche, um Verbesserungs- } \\
\text { potenziale zu bestimmen }\end{array}$ & $40 \%$ & $24 \%$ & $47 \%$ & $59 \%$ & $13 \%$ & $17 \%$ \\
\hline $\begin{array}{l}\text { Verwarnungen bei Nicht-Ein- } \\
\text { haltung von Forderungen }\end{array}$ & $53 \%$ & $41 \%$ & $34 \%$ & $35 \%$ & $13 \%$ & $24 \%$ \\
\hline $\begin{array}{l}\text { Beendigung der Lieferantenbe- } \\
\text { ziehung }\end{array}$ & $20 \%$ & $29 \%$ & $60 \%$ & $41 \%$ & $20 \%$ & $30 \%$ \\
\hline $\begin{array}{l}\text { Schulungen für Verbesserung } \\
\text { von Sozial- und/oder Umwelt- } \\
\text { bedingungen }\end{array}$ & $13 \%$ & $6 \%$ & $67 \%$ & $47 \%$ & $20 \%$ & $47 \%$ \\
\hline
\end{tabular}

Tabelle 3: Massnahmen, um Umwelt-/Sozialleistungen bei Lieferanten zu verbessern 


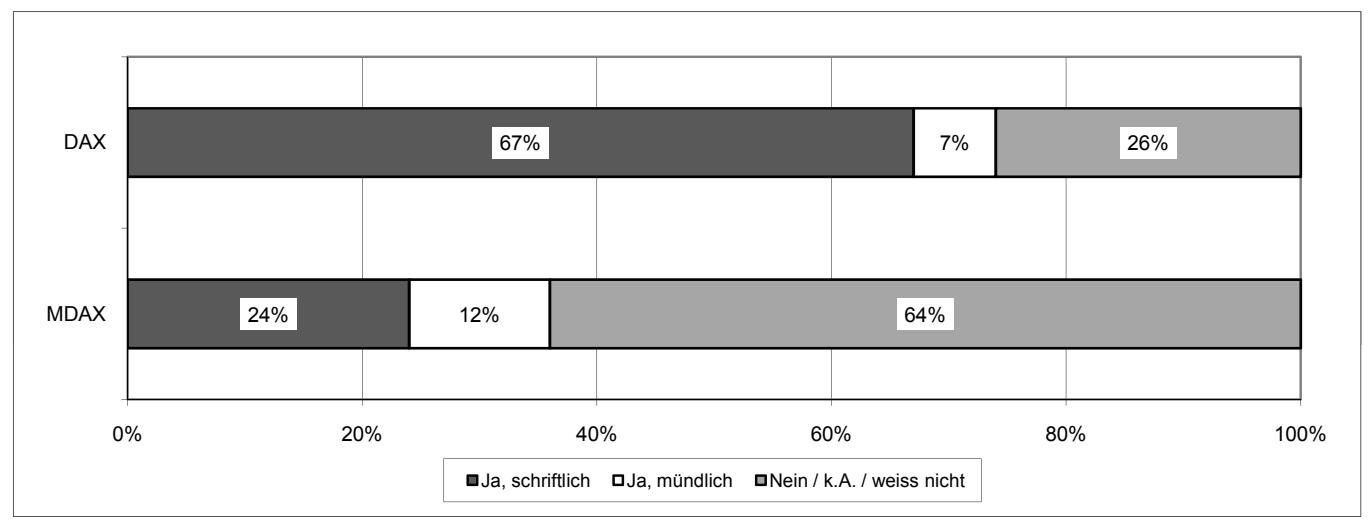

Abbildung 4: Festschreibung von Sanktionsmöglichkeiten in Lieferantenvereinbarungen

Unter einer zusätzlichen offenen Frage geben Unternehmen an, dass verschiedene Massnahmen (z. B. Schulungen; schriftliche Verwarnungen und Auftragskürzungen) einer Beendigung der Geschäftsbeziehung vorgezogen werden. Die Auslistung von Lieferanten ist also tendenziell anderen Massnahmen nachgestellt. Andere empirische Studien zeigen ein ähnliches Bild: Verstösse gegen Standards werden in manchen Branchen eher als Regel denn als Ausnahme identifiziert (Peters 2010, 50 f.; PwC/Oekom Research 2009), so dass auch eine Beendigung der Lieferantenbeziehung nur begrenzt als praktikabel angesehen wird. Ausserdem wird beobachtet, dass Anforderungen in Bezug auf Standards und Codes of Conduct nur als Ausgangspunkt für einen kollaborativen Entwicklungsprozesses gelten und somit die harte Sanktionierung durch Beziehungsabbruch im Hintergrund steht ( $M a$ mic 2005; Bernstein/Greenwald 2009). Auch Holt (2004) berichtet davon, dass Lieferanten selten mit einem Beziehungsabbruch konfrontiert sind. Dies bedeutet, dass ein Lieferantenwechsel derzeit hohe Wechselkosten verursacht und keinen entscheidenden Gewinn an Information und Sicherheit bezüglich ökologischer und sozialer Aspekte bringt, während die Lieferantenentwicklung als ökonomisch sinnvoller bewertet wird, da die Kombination aus Schulungskosten und zusätzlichem Informations- und Sicherheitsnutzen höher eingestuft wird.

\section{Diskussion und Forschungsbedarf}

\subsection{Unternehmensgrösse und Supply Chain Charakteristika}

Die empirische Untersuchung der DAX- und MDAX-Unternehmen zeigt, dass die Unternehmensgrösse für die Ausgestaltung des SSCM Bedeutung hat, insbesondere im Sinne einer höheren Formalisierung der Massnahmen. Sie bestätigt damit Ergebnisse bisheriger Studien zur Rolle der Unternehmensgrösse für das SSCM (Delmas/Terlaak 2001; Neilson/ Pritchard 2007; Peters 2010). Vor dem Hintergrund, dass die bisherigen Studien z.T. Grossunternehmen mit KMUs verglichen haben, ist es bemerkenswert, wie stark der Unterschied bereits zwischen börsennotierten Grossunternehmen des DAX und den nächstgrossen Unternehmen des MDAX ausfällt. Dies kann zwei Ursachen haben: Erstens scheint zusätzlich zu der Rolle der Unternehmensgrösse, die (im Mittel) bei DAX-Unternehmen grösser als bei MDAX ist, die Bedeutung der (nach Marktkapitalisierung vorge- 
nommenen) Zuteilung zu Large Cap (DAX) oder Mid Cap (MDAX) für eine höhere Exponiertheit (vgl. auch Peters 2010, 61) in der Öffentlichkeit ausschlaggebend zu sein. Die mediale Aufmerksamkeit konzentriert sich danach insbesondere auf die „führende“ Gruppe der Large Caps. Auch internationale Studien im SSCM zeigen wesentliche Unterschiede zwischen Mid und Large Caps (Bernstein/Greenwald 2009). Zweitens bedingt die Unternehmensgrösse auch die Charakteristika der Supply Chain selbst. So haben die DAX-Unternehmen im Vergleich zu MDAX-Unternehmen sowohl knapp doppelt so häufig Lieferantenbeziehungen mit mehr als 5.000 Lieferanten als auch mehr als 50 Beschaffungsländer sowie eine wesentlich höhere Beschaffungsquote aus Entwicklungs- und Schwellenländern. Dieser höheren Komplexität versuchen die DAX-Unternehmen mit formalisierteren Managementmassnahmen zu begegnen. Da grössere Unternehmen i.d.R. auch über eine umfangreichere Ressourcenausstattung verfügen, können sie diese Massnahmen auch eher realisieren (Neilson/Pritchard 2007). Darüber hinaus ist zu bedenken, dass sich die offenkundige Komplexität in internationalen Lieferantenbeziehungen noch weiter durch die Berücksichtigung von Umwelt- und Sozialaspekten in der Wertschöpfungskette erhöht.

Parallel zu diesen Ergebnissen hinsichtlich der Unternehmensgrösse lassen sich verschiedene Entwicklungen hinsichtlich der konkreten Massnahmen im Lieferantenmanagement aufzeigen.

\subsection{Anforderungen, Bewertung und Auswahl}

Zur Festschreibung ökologischer und sozialer Aspekte in Vereinbarungen und Verträgen setzen die DAX- und MDAX-Unternehmen am stärksten auf Signalling der Lieferanten hinsichtlich der vom Unternehmen erwarteten Mindeststandards (vgl. Tabelle 2). Am zweithäufigsten werden Anforderungen in Lieferverträgen festgeschrieben und somit ein Interessenausgleich zwischen fokalem Unternehmen und Lieferanten durch mögliche Sanktionsmöglichkeiten geschaffen. Die drittwichtigste Gruppe beinhaltet die Bewertungs- und Auditierungs-Mechanismen, die bei Anwendung vor Vertragsabschluss einer Adverse Selection entgegen wirken sollen sowie bei Nutzung nach Vertragsabschluss der Verhinderung des Moral Hazards dienen. Insgesamt werden also zum Abbau von Informationsasymmetrien bezüglich ökologischer/sozialer Aspekte eher auf leicht zu implementierende Mechanismen zurückgegriffen (Mindeststandards, Vertragsinklusion) als auf ressourcenaufwendige Screening- und Kontroll-Mechanismen. Nachhaltigkeitskriterien bleiben also häufiger auf dem ersten oder zweiten Niveau der (wenn auch verbindlichen) Anforderung. Die Anforderungen werden allerdings nicht immer mit konkreten Kontroll- bzw. Auditierungsmechanismen unterlegt. Dies könnte ein Zeichen für eine oberflächliche sowie auf Reputation ausgerichtete Umsetzung sein (Müller 2005, 88).

Mit Blick auf die Anforderungen der Unternehmen zeigt sich, dass bei einer entsprechenden schriftlichen Fixierung eine Tendenz zugunsten ökologischer Themen gegenüber den sozialen beobachtet werden kann, obwohl Unternehmen die Bedeutung der Themen weitgehend gleichmässig einschätzen (vgl. Abschnitt 4.2.1). Die rund ein Jahrzehnt frühere Betonung ökologischer Themen (1980er Jahre) im Vergleich zu den sozialen Themen (1990er Jahre) mag noch heute eine Rolle für die Formulierung von Anforderungen spielen (Andersen 2005, 67). Die grundsätzlich bessere Messbarkeit - und damit bessere Kontrollmöglichkeiten - von ökologischen Aspekten kann ein weiterer Grund hierfür sein ( $R i$ chards/Gladwin 1999; Schaltegger et al. 2007). 


\subsection{Lieferantenentwicklung}

Die Untersuchung zeigt, dass zur Verbesserung der Nachhaltigkeitsleistung von Lieferanten Vor-Ort-Kontrollen, Verwarnung, Potenzialgespräche sowie Schulungs- und Entwicklungsmassnahmen genutzt werden (vgl. Tabelle 3). Auch die Beendigung einer Lieferantenbeziehung wird als letztes Mittel wahrgenommen. Diese ist allerdings weder eine wirksame noch effiziente Pauschallösung, ist sie doch mit hohen Transaktionskosten (z. B. Such- und Vereinbarungskosten) verbunden. Noch wichtiger als die Rolle der Transaktionskosten erscheint jedoch, dass der Markt für Lieferanten, die umfangreiche Nachhaltigkeitskriterien erfüllen können, viel knapper ist als für solche, die nur konventionelle Lieferkriterien erfüllen (z. B. Qualität etc.; Reuter et al. 2010). Ausserdem besteht bei neuen Lieferanten keine Sicherheit, dass diese tatsächlich nachhaltig im Sinne der Anforderungen des fokalen Unternehmens handeln. In diesem Kontext erscheinen alternative Massnahmen und dabei insbesondere die Lieferantenentwicklung von besonderem Interesse.

Im Unterschied zu älteren Studien (z. B. Holt 2004), aber in Einklang mit anderen aktuellen empirischen Untersuchungen (Mamic 2005; Reuter et al. 2010) lässt sich auch in dieser Analyse eine hohe Bedeutung der Lieferantenentwicklung feststellen. Insbesondere bei den sehr stark internationalisierten Supply Chains der DAX-Konzerne und der grossen Relevanz von Entwicklungs- und Schwellenländern spielt die Lieferantenentwicklung für den Know-how-Aufbau eine wichtige Rolle.

Der Bedeutungsgewinn der nachhaltigkeitsorientierten Lieferantenentwicklung ist aus mindestens zwei Gründen wünschenswert: Erstens werden im entgegengesetzten Fall der Lieferantenaufkündigung soziale oder ökologische Missstände nicht aufgehoben, sondern ggf. sogar zementiert, da sich Lieferanten durch ökonomische Einbussen gezwungen sehen können, weiter vornehmlich auf kurzfristige Kostenaspekte zu schauen. Zweitens führt die Lieferantenentwicklung zu erwünschten Spillover-Effekten, so dass die nachhaltigkeitsorientierte Entwicklung der Lieferanten auch bei deren Vorlieferanten und in anderen Wertschöpfungsketten, in denen die Lieferanten beteiligt sind, hineinwirkt. Lieferantenentwicklung gibt daher Impulse für die Verbesserung der Nachhaltigkeitsleistung in ganzen Regionen und Branchen.

Die Lieferantenentwicklung ist jedoch nicht ganz unproblematisch und sollte gut gemanagt werden. Im Unterschied zu Adverse Selection- und Moral Hazard-Problemen spielt bei der Lieferantenentwicklung eher die Hold-up-Problematik (Gefahr einer Ressourcenabhängigkeit des fokalen Unternehmens durch getätigte Investitionen bei Lieferanten) eine Rolle. Bei einem Wegfall des Lieferanten (z. B. durch opportunistisches Verhalten des Lieferanten, der sich durch verbesserte Leistungsfähigkeit auch anderen Kunden zuwenden kann; vgl. Holt 2004) stellen bisher investierte Massnahmen wie Schulungen etc. verlorene Kosten („Sunk costs“) dar. Unternehmen sollten daher anreizkompatible Verträge (z. B. langfristige Lieferverträge, strategische Partnerschaften, Lieferantenintegration) ausgestalten, um die Investitionen in die Lieferantenentwicklung abzusichern.

Letztendlich kann eine Lieferantenentwicklung die Basis für eine angestrebte längerfristige Zusammenarbeit zwischen Unternehmen und Lieferanten sein. Dadurch wird es wiederum möglich, dass einem raschen Wechsel von Lieferantenbeziehungen durch Ausnutzen von Standortvorteilen entgegengewirkt wird - da der Wechsel z. B. für soziale Aspekte wie Arbeitsbedingungen oder langfristige Beschäftigung kritisch ist. Müssen nämlich neue Lieferanten an anderen Standorten und in weniger entwickelten Ländern wieder neu mit sozialer und ökologischer Expertise aufgebaut werden, wird ein schneller Lieferantenwechsel 
weniger interessant. Der Vertrauensaufbau zwischen Unternehmen und Lieferanten einerseits und die Reduzierung von Lieferantenwechseln andererseits vermindern schliesslich Transaktionskosten und können sich so auch ökonomisch positiv auf die Kostenstruktur des fokalen Unternehmens auswirken.

\subsection{Forschungsbedarf und Limitationen}

Die steigende Bedeutung der Kooperation mit Lieferanten sollte auch in der weiteren Forschungsagenda berücksichtigt werden. Drei Strategien sind denkbar:

- Mögliche Gründe, weshalb die weniger grossen Unternehmen (MDAX) insgesamt weniger Umsetzungsstärke im SSCM zeigen, können hohe Kosten für Informationsbeschaffung und Kommunikation sowie Monitoring und Entwicklung sein. Des Weiteren können Spillover-Effekte eine Rolle spielen, wo andere Unternehmen (und vielleicht Wettbewerber) von den jeweiligen Massnahmen bei Lieferanten profitieren, ohne selbst zu investieren. Daher sind interorganisationale Ansätze wie horizontale Einkaufskooperationen oder -netzwerke (Picot et al. 2003, 306; Holt 2004) zu untersuchen.

- Die empirische Untersuchung bestätigt die hohe strategische Bedeutung der Beschaffungsabteilung für die Umsetzung von Nachhaltigkeit in Unternehmen (Günther et al. 2005, 8; Preuss 2007). Zusätzlich ist sie herausgefordert, neben dem konventionellen Know-how des Einkaufs zusammen mit anderen Funktionsbereichen soziale und ökologische Expertise auf- und auszubauen. Zukünftige Forschung sollte daher Ansätze untersuchen, die eine intensivierte funktionsübergreifende Zusammenarbeit ermöglichen, wie z. B. sog. „1:1 Gespräche“ zwischen Abteilungen (Hansen 2010, 215).

- Die enge Lieferantenkooperation kann auch als Basis für kollaborative Entwicklungsprozesse von nachhaltigkeitsorientierten Produkten dienen - denn diese erfordern eine Betrachtung ökologischer und sozialer Aspekte über den gesamten physischen Lebenszyklus (Preuss 2007; Vachon 2007; Seuring/Müller 2008; Wagner 2008; Hansen et al. 2009). Eine enge Zusammenarbeit im Rahmen der Lieferantenentwicklung kann eine gute Ausgangsbasis für solche F\&E-Kooperationen in der Supply Chain sein.

Bei der Interpretation der Ergebnisse ist zu beachten, dass die empirische Untersuchung auf grundlegenden statistischen Analysen (insb. Häufigkeiten) aufbaut, die keine prognostische Wirkung haben. Eine Generalisierung der Ergebnisse ist auch aufgrund der kleinen Anzahl der Unternehmen in den Untersuchungsgruppen DAX und MDAX (trotz der sehr hohen Rücklaufquote) eingeschränkt. Weiterhin unterliegt die Studie durch den Fokus auf soziale und ökologische Themen wie andere Studien in diesem Bereich dem Störfaktor der sozialen Erwünschtheit (Fernandes/Randall 1992). Durch die anonymisierte Behandlung der Unternehmen sowie durch das spezifische Design des Fragebogens (z. B. notwendige Angabe von Beispielen) wurde dieser so weit wie möglich eingeschränkt. Zukünftige Studien könnten über die Selbstauskünfte der Unternehmen hinaus eine direkte Befragung von Lieferanten und kritischen Stakeholdern vornehmen.

\section{Fazit und Ausblick}

SSCM befasst sich mit der Gestaltung von Wertschöpfungsketten unter ökonomischen, ökologischen und sozialen Gesichtspunkten. Insbesondere durch die hohe Internationalisierung der Wertschöpfungsketten und der Beschaffung aus Entwicklungs- und Schwellenländern erlangt das SSCM sowohl in der Wissenschaft als auch der Praxis eine steigende 
Bedeutung. Das Lieferantenmanagement ist dabei von zentraler Bedeutung für die Umsetzung von Nachhaltigkeitsaspekten in der Lieferkette. Die durchgeführte Unternehmensbefragung bei DAX- und MDAX-Unternehmen zeigt, dass bei einem Grossteil der an der Befragung teilnehmenden Unternehmen ökologische und/oder soziale Aspekte im Lieferantenmanagement des Unternehmens integriert werden. Dabei unterscheiden sich DAX- und MDAX-Unternehmen in der Ausgestaltung des SSCM.

Entwicklungsbedarf besteht in Bezug auf die Ausweitung des Themenfokus des SSCM von den direkt mit Produktionsprozessen in Verbindung stehenden Themen auf indirekt wirkende Themen wie Artenvielfalt oder Arbeitsplatzsicherheit. Bezüglich der Massnahmen ist eine Entwicklung von Kontroll- und Überwachungsansätzen hin zu Ansätzen einer partnerschaftlichen Lieferantenentwicklung zu erkennen und erforderlich. Vor dem Hintergrund, dass die Kontrollkosten sehr hoch sind, die Nachhaltigkeitsrisiken entlang der Lieferkette nur beschränkt reduziert werden können und der Markt für Lieferanten mit hohen Sozial- und Ökostandards begrenzt ist, sind Massnahmen zur Lieferantenentwicklung ökonomisch sinnvoll. Lieferantenentwicklungen sind auch für die (soziale und ökologische) Nachhaltigkeit wünschenswert, da durch Spillover-Effekte weitergehende Entwicklungsprozesse angestossen werden. Die Lieferantenentwicklung ist angesichts der Hold-upProblematik jedoch mit anreizkompatiblen Verträgen auszugestalten oder durch Unternehmenskooperationen zu realisieren.

Zukünftige Forschung sollte ein besonderes Augenmerk auf kooperative Lernprozesse in der Supply Chain legen, da Kooperation für die Realisierung von nachhaltigkeitsorientierten Geschäftsprozessen und Innovationen einen zentralen Faktor darstellt.

\section{Anhang (Auszug aus dem Fragebogen)}

\begin{tabular}{ll}
\hline Referenz & Fragen \\
\hline Abb. 1: & $\begin{array}{l}\text { Wenn Sie Ihre internationalen Lieferantenbeziehungen betrachten, wie ausschlaggebend sind fol- } \\
\text { gende Gründe für die Komplexität Ihrer Lieferantenbeziehungen? (Auf der Skala: Überhaupt } \\
\text { nicht ausschlaggebend / Wenig ausschlaggebend / Ausschlaggebend / Sebr ausschlaggebend / } \\
\text { Weiss nicht) }\end{array}$ \\
\hline Abb. 2: & $\begin{array}{l}\text { Wer in Ihrem Unternehmen verlangt die Berücksichtigung von ökologischen und/oder sozialen } \\
\text { Aspekten (z. B. die Verminderung von Emissionen, Anti-Diskriminierung, Reduzierung von Ge- } \\
\text { sundheits- und Unfallgefahren) in Ihrem Supply Chain Management durch schriftliche oder } \\
\text { mündliche Äusserungen? (Mehrfachnennungen möglich) }\end{array}$ \\
\hline
\end{tabular}

Tab. 2: $\quad$ Sind ökologische / soziale Anforderungen (wie z. B. die Einhaltung von Umweltstandards / Einhaltung von Unfallschutzmassnahmen) in Verträgen bzw. Vereinbarungen mit Ihren Lieferanten explizit schriftlich festgehalten? (Nein, da nicht relevant / Nein, jedoch geplant / Nein, jedoch mündlich / Ja, schriftlich / Weiss nicht)

Abb. 3:

Bei wie vielen Lieferanten Ihres Unternehmens verlangen Sie den Nachweis über folgende Qualitäts-, Umwelt- und/oder Sozialstandards (inkl. Audits)? (Keine Lieferanten / Wenige Lieferanten / Viele bis alle Lieferanten / Weiss nicht)

Tab. 3: Wie häufig ergreifen Sie folgende weitere Massnahmen, um Sozial- und/oder Umweltleistungen bei Ihren Lieferanten zu verbessern? (Nie / Manchmal / Immer / Weiss nicht)

Abb. 4:

Haben Sie bei Nichteinhaltung von ökologischen und/oder sozialen Vorgaben in Ihren Lieferantenvereinbarungen Sanktionsmöglichkeiten festgeschrieben? (Ja, schriftlich / Ja, mündlich / Nein; Wenn ja, welche Sanktionsmassnahmen haben Sie gegenüber Ihren Lieferanten bzw. Vorlieferanten schon durchsetzt)

Tabelle 4: Auszug aus dem Fragebogen 


\section{Literaturhinweise}

Andersen, M. (2005): Corporate Social Responsibility in Global Supply Chains - Understanding the uniqueness of firm behaviour, $\mathrm{PhD}$ Series 15.2005, Kopenhagen.

Arnold, U./Eßig, M. (2002): Grundlagen des internationalen Supply Chain Managements, in: Macharzina, K./Oesterle, M.-J. (Hrsg.): Handbuch Internationales Management. Grundlagen, Instrumente, Perspektiven, 2. überarb. und erw. Aufl., Wiesbaden, S. 237-254.

Bai, C./Sarkis, J. (2010): Green Supplier Development - Analytical Evaluation Using Rough Set Theory, in: Journal of Cleaner Production, Vol. 18, No. 12, S. 1200-1210.

Bernstein, A./Greenwald, C. (2009): Benchmarking Corporate Policies on Labor and Human Rights in Global Supply Chains, in: Occasional Paper Series, No. 5, Harvard.

Beske, P., et al. (2008): The Use of Environmental and Social Standards by German First-Tier Suppliers of the Volkswagen AG, in: Corporate Social Responsibility and Environmental Management, Vol. 15, No. 2, S. 63-75.

BMU (Bundesministerium für Umwelt, Naturschutz und Reaktorsicherheit) (2007): Nationale Strategie zur biologischen Vielfalt, Berlin.

BMU (Bundesministerium für Umwelt Naturschutz und Reaktorsicherheit) (2008): Umweltbewusstsein in Deutschland 2008. Ergebnisse einer repräsentativen Bevölkerungsumfrage, Berlin.

Bruhn, M./Meffert, H. (2006): Umweltbewusstsein der Bevölkerung in der Bundesrepublik Deutschland - empirische Ergebnisse einer Langzeitstudie, in: Die Unternehmung, Jg. 60, Nr. 1, S. 7-26.

Carter, C.R./Dresner, M. (2001): Purchasing's Role in Environmental Management. Cross-Functional Development of Grounded Theory, in: The Journal of Supply Chain Management, Vol. 37, No. 3, S. 12-27.

Carter, C.R., et al. (1998): Environmental Purchasing. Benchmarking Our German Counterparts, in: International Journal of Purchasing and Materials Management, Vol. 34, No. 4, S. 28-38.

Carter, C.R./Jennings, M.M. (2004): The Role of Purchasing in Corporate Social Responsibility - A Structural Equation Analysis, in: Journal of Business Logistics, Vol. 25, No. 1, S. 145-186.

Chien, M.K./Shih, L.H. (2007): An empirical study of the implementation of green supply chain management practices in the electrical and electronic industry and their relation to organizational performances, in: International Journal of Environmental Science and Technology, Vol. 4, No. 3, S. 383-394.

Coase, R.H. (1937): The Nature of the Firm, in: Economica, Vol. 4, No. 16, S. 386-405.

Cooper, M.C., et al. (1997): Supply Chain Management. More Than a New Name for Logistics, in: International Journal of Logistics Management, Vol. 8, No. 1, S. 1-14.

Darnall, N./Carmin, J. (2005): Greener and cleaner? The signaling accuracy of U.S. voluntary environmental programs, in: Policy Sciences, Vol. 38, No. 2-3, S. 71-90.

Delmas, M.A./Montiel, I. (2009): Greening the Supply Chain. When Is Customer Pressure Effective?, in: Journal of Economics \& Management Strategy, Vol. 18, No. 1, S. 171-201.

Delmas, M.A./Terlaak, A.K. (2001): A framework for analyzing environmental voluntary agreements, in: California Management Review, Vol. 43, No. 3, S. 44-63.

De Nardo, M., et al. (2010): Global Sourcing Footprint. Eine Portfolio-Methodik zur Unterstützung der systematischen Entscheidungsfindung in der Beschaffung, in: Bogaschewsky, R., et al. (Hrsg.): Supply Management Research. Aktuelle Forschungsergebnisse 2010, Wiesbaden, S. 221246.

Deutsche Börse (2007): Die Indexwelt der deutschen Börse, Frankfurt/Main. 
Elkington J. (1999): Cannibals with forks. The triple bottom line of the 21st century, Oxford.

Fernandes, M.F./Randall, D.M. (1992): The nature of social desirability response effects in ethics research, in: Business Ethics Quarterly, Vol. 2, No. 2, S. 183-205.

Gießmann, M./Lasch, R. (2010): Der Einfluss der Beschaffungskomplexität auf den Logistikerfolg. Eine kausalanalytische Untersuchung unter Verwendung des Partial-Least-Squares, in: Bogaschewsky, R., et al. (Hrsg.): Supply Management Research. Aktuelle Forschungsergebnisse 2010, Wiesbaden, S. 149-196.

Gomm, M. (2008): Supply-chain-Finanzierung. Optimierung der Finanzflüsse in Wertschöpfungsketten, Berlin.

Gold, S., et al. (2010): Sustainable Supply Chain Management and Inter-Organizational Resources. A Literature Review, in: Corporate Social Responsibility and Environmental Management, Vol. 17, No. 4, S. 230-245.

Goldbach, M. (2001): Akteursbeziehungen in nachhaltigen Wertschöpfungsketten, EcoMTex-Diskussionspapier, 3, Oldenburg.

Goldbach, M. (2003): Koordination von Wertschöpfungsketten durch Target Costing und Öko-Target Costing. Eine agentur- und strukturationstheoretische Reflexion, Wiesbaden.

GRI (2006): Global Reporting Initiative - Leitfaden zur Nachhaltigkeitsberichterstattung, Version 3.0, unter http://www.globalreporting.org/NR/rdonlyres/B77474D4-61E2-4493-8ED0-D4AA9BEC000D/2868/G3_LeitfadenDE1.pdf, Abruf: 6.8.2010.

Günther, E., et al. (2005): Green eBusiness. Auswertung der empirischen Untersuchung zu Hemmnissen umweltfreundlicher Beschaffung, Dresdner Beiträge zur Betriebswirtschaftslehre, 102/05, Dresden.

Hall, J. (2000): Environmental supply chain dynamics, in: Journal of Cleaner Production, Vol. 8, No. 6, S. 455-471.

Handfield, R.B., et al. (2002): Applying environmental criteria to supplier assessment. A study in the application of the analytical hierarchy process, in: European Journal of Operational Research, Vol. 141, No. 1, S. 70-87.

Hansen, E.G. (2010): Responsible Leadership Systems. An Empirical Analysis of Integrating Corporate Responsibility into Leadership Systems, Wiesbaden.

Hansen, E.G., et al. (2009): Sustainability Innovation Cube - A Framework to Evaluate Sustainability-Oriented Innovations, in: International Journal of Innovation Management, Vol. 13, No. 4, S. 683-713.

Hofman, E./Westerfeld, S. (2010): Bestandsfinanzierung in Supply Chains durch Logistikunternehmen, in: Die Unternehmung, Jg. 64, Nr. 3, S. 291-312.

Holt, D. (2004): Managing the interface between suppliers and organizations for environmental responsibility - an exploration of current practices in the UK, in: Corporate Social Responsibility and Environmental Management, Vol. 11, No. 2, S. 71-84.

Jahns, C., et al. (2007): The Quest for Competitive Advantage. Empirical Evidence of the Role of Resources in a Global Sourcing Context, in: Die Unternehmung, Jg. 61, Nr. 3, S. 213-226.

Klassen, R.D./Vachon, S. (2003): Collaboration and evaluation in the supply chain. The impact on plant-level environmental investment, in: Production and Operations Management, Vol. 12, No. 3, S. 336-352.

Kolk, A., et al. (1999): International codes of conduct and corporate social responsibility. Can transnational corporations regulate themselves?, in: Transnational Corporations, Vol. 8, No. 1, S. 143-181. 
Koplin, J. (2006): Nachhaltigkeit im Beschaffungsmanagement. Ein Konzept zur Integration von Umwelt- und Sozialstandards, Wiesbaden.

Koplin, J., et al. (2007): Incorporating sustainability into supply management in the automotive industry - The case of the Volkswagen AG, in: Journal of Cleaner Production, Vol. 15, No. 11, S. 1053-1062.

Kotzab, H.W., et al. (2006): The Implementation of Supply Chain Management within Organizations-Construct. Measurement and Explorative Empirical Discussion, in: Die Unternehmung, Jg. 60, Nr. 2, S. 89-104.

Kumar, S./Malegeant, P. (2006): Strategic alliance in a closed-loop supply chain. A case of manufacturer and eco-nonprofit organization, in: Technovation, Vol. 26, No. 10, S. 1127-1135.

Lamming, R./Hampson, J. (1996): The Environment as a Supply Chain Management Issues, in: British Journal of Management, Vol. 7, Special Issue, S. S45-S62.

Locke, R. (2003): The Promise and Perils of Globalization. The Case of Nike, in: Kochan, T./ Schmalensee, R. (Hrsg.): Management. Inventing and delivering its future, Cambridge, S. 39-70.

Loew, T. (2006): CSR in der Supply Chain. Herausforderungen und Ansatzpunkte für Unternehmen, Berlin, unter http://www.4sustainability.org/seiten/csr-publikationen.htm, Abruf: 2.8.2010.

Mamic, I. (2005): Managing Global Supply Chain. The Sports Footwear, Apparel and Retail Sectors, in: Journal of Business Ethics, Vol. 59, No. 1-2, S. 81-100.

Min, H./Galle, W.P. (2001): Green purchasing practices of US firms, in: International Journal of Operations \& Production Management, Vol. 21, No. 9, S. 1222-1238.

Müller, M. (2005): Informationstransfer im Supply Chain Management. Analyse aus der Neuen Institutionenökonomie, Wiesbaden.

Müller, M., et al. (2009): The Contribution of Environmental and Social Standards. Towards Ensuring Legitimacy in Supply Chain Governance, in: Journal of Business Ethics, Vol. 89, No. 4, S. 509-523.

Neilson, J./Pritchard, B. (2007): Green coffee? The contradictions of global sustainability initiatives from an Indian perspective, in: Development Policy Review, Vol. 25, No. 3, S. 311-331.

New, S., et al. (2000): Buying the environment - The multiple meanings of green supply, in: Fineman, S. (Hrsg.): The Business of Greening, London, S. 33-53.

Pagell, M./Wu, Z. (2009): Building a more complete theory of sustainable supply chain management using case studies of 10 exemplars, in: Journal of supply chain management, Vol. 45, No. 2, S. 37-56.

Peters, N. (2010): Inter-organisational design of voluntary sustainability initiatives. Increasing the legitimacy of sustainability strategies for supply chains, Wiesbaden.

Picot, A., et al. (2003): Die grenzenlose Unternehmung. Information Organisation und Management. Lehrbuch zur Unternehmensführung im Informationszeitalter, 5., aktualisierte Aufl., Wiesbaden.

Preuss, L. (2001): In dirty chains? Purchasing and greener manufacturing, in: Journal of Business Ethics, Vol. 34, No. 3-4, S. 345-359.

Preuss, L. (2007): Contribution of purchasing and supply management to ecological innovation, in: International Journal of Innovation Management, Vol. 11, No. 4, S. 515-537.

PricewaterhouseCoopers (PwC)/Oekom Research (Hrsg.) (2009): Corporate Responsibility bei Auslandsinvestitionen, Frankfurt am Main, unter www.oekom-research.de/homepage/german/ oekom_PwC_Auslandsinvestitionen.pdf, Abruf: 9.8.2010. 
Reuter, C., et al. (2010): Sustainable Global Supplier Management - The Role of Dynamic Capabilities in Achieving Competitive Advantage, in: Journal of Supply Chain Management, Vol. 46, No. 2-3, S. 45-63.

Richards, D.J./Gladwin, T.N. (1999): Sustainability metrics for the business enterprise, in: Environmental Quality Management, Vol. 8, No. 3, S.11-21.

Schaltegger, S./Burritt, R. (2005): Corporate Sustainability, in: Folmer, H./Tietenberg, T. (Eds.): The International Yearbook of Environmental and Resource Economics 2005/2006. A Survey of Current Issues, Cheltenham, S. 185-222.

Schaltegger, S., et al. (2007): Nachhaltigkeitsmanagement in Unternehmen. Von der Idee zur Praxis. Managementansätze zur Umsetzung von Corporate Social Responsibility und Corporate Sustainability, 3. Aufl., Berlin/Lüneburg.

Schaltegger, S./Wagner, M. (2006): Integrative management of sustainability performance, measurement and reporting, in: International Journal of Accounting, Auditing and Performance Evaluation, Vol. 3, No. 3, S. 1-19.

Schaltegger, S., et al. (2010): Corporate Sustainability Barometer 2010. Wie nachhaltig agieren Unternehmen in Deutschland?, Frankfurt a.M./Lüneburg.

Seuring, S., et al. (2004): Managing time and complexity in supply chains - two cases from the textile industry, in: International Journal of Integrated Supply Management, Vol. 1, No. 2, S. 180198.

Seuring, S./Müller, M. (2007): Integrated Chain Management in Germany - Identifying Schools of Thought Based on a Literature Review, in: Journal of Cleaner Production, Vol. 15, No. 7, S. 699-710.

Seuring, S./Müller, M. (2008): From a literature review to a conceptual framework for sustainable supply chain management, in: Journal of Cleaner Production, Vol. 16, No. 15, S. 1699-1710.

Sharma, S./Vredenburg, H. (1998): Proactive corporate environmental strategy and the development of competitively valuable organizational capabilities, in: Strategic Management Journal, Vol. 19, No. 8, S. 729-754.

Simpson, D., et al. (2007): Greening the automotive supply chain. A relationship perspective, in: International Journal of Operations \& Production Management, Vol. 27, No. 1, S. 28-48.

Stölzle, W./Heusler, K.F. (2004): Implementierung von Supply Chain Management - Ressourcenorientierte Ableitung eines konzeptimmanenten Kompetenzprofils, in: Eßig, M. (Hrsg.): Perspektiven des Supply Management - Konzept und Anwendungen, Berlin, S. 199-233.

Stölzle, W./Lukas, U. (2007): Grüne Lösungen für schwarze Zahlen. Universität St. Gallen und DKV prämieren nachhaltige Transportkonzepte, in: DVZ, Jg. 61, Nr. 98, S. 11.

Teuscher P., et al. (2006): Risk management in sustainable supply chain management (SSCM). Lessons learnt from the case of GMO-free soybeans, in: Corporate Social Responsibility and Environmental Management, Vol. 13, No. 1, S. 1-10.

Teuteberg, F./Wittstruck, D. (2010): A Systematic Review of Sustainable Supply Chain Management Research. What is there and what is missing?, in: Schumann, M., et al. (Hrsg.): Tagungsband zur Multikonferenz Wirtschaftsinformatik 2010, Göttingen, S. 1001-1015.

Vachon S. (2007): Green supply chain practices and the selection of environmental technologies, in: International Journal of Production Research, Vol. 45, No. 18/19, S. 4357-4379.

Vachon, S., et al. (2009): Aligning competitive priorities in the supply chain. The role of interactions with suppliers, in: International Journal of Operations \& Production Management, Vol. 29, No. 4, S. 322-340. 
Wagner, M. (2008): Die Funktion von Anspruchsgruppen bei Kooperationen in der Produktentwicklung. Eine empirische Analyse im deutschen Verarbeitenden Gewerbe, in: Die Unternehmung, Jg. 62, Nr. 6, S. 521-541.

Walker, H., et al. (2008): Drivers and barriers to environmental supply chain management practices. Lessons from the public and private sector, in: Journal of Purchasing \& Supply Management, Vol. 14, No. 1, S. 69-85.

Walton, S.V., et al. (1998): The green supply chain: Integrating suppliers into environmental management processes, in: International Journal of Purchasing and Materials Management, Vol. 34, No. 2, S. 2-11.

Werner, H. (2008): Supply Chain Management. Grundlagen, Strategien, Instrumente und Controlling, 3. Aufl., Wiesbaden.

Wildemann, H. (2000): Supply Chain Management, München.

Williamson, O.E. (1975): Markets and hierarchies. Analysis and antitrust implications. A study in the economics of internal organization, New York.

Winkler, H., et al. (2007): Entwicklung eines Performance- und Risikomanagement-Konzeptes für nachhaltige Supply Chain Netzwerke. Ein Projektbericht im Rahmen der Programmlinie Fabrik der Zukunft, Berichte aus Energie- und Umweltforschung, 19/2007, Wien.

Wittstruck, D./Teuteberg, F. (2010 a): Ein Referenzmodell für das Sustainable Supply Chain Management, in: Zeitschrift für Management, Jg. 5, Nr. 2, S. 141-164.

Wittstruck, D./Teuteberg, F. (2010 b): Sustainable Supply Chain Management in Recyclingnetzwerken der Elektro- und Elektronikindustrie. Eine empirische Studie zum Status Quo, in: Schumann, M., et al. (Hrsg.): Tagungsband zur Multikonferenz Wirtschaftsinformatik 2010, Göttingen, S. 1029-1043.

Wycherley, I. (1999): Greening supply chains - The case of Body Shop International, in: Business Strategy and the Environment, Vol. 8, No. 2, S. 120-127.

Zadek, S. (2004): The Path to Corporate Responsibility, in: Harvard Business Review, Vol. 82, No. 12, S. 125-132.

Zhu, Q.H./Sarkis, J. (2006): An inter-sectoral comparison of green supply chain management in China. Drivers and practice, in: Journal of Cleaner Production, Vol. 14, No. 5, S. 471-486.

Zsidisin, G.A./Siferd, S.P. (2001): Environmental purchasing. A framework for theory development, in: European Journal of Purchasing \& Supply Management, Vol. 7, No. 1, S. 61-73. 
Erik G. Hansen, Dr., ist als wissenschaftlicher Assistent am Centre for Sustainability Management (CSM) der Leuphana Universität und als Visiting Fellow am Doughty Centre for Corporate Responsibility der Cranfield University, UK tätig.

Dorli Harms ist wissenschaftliche Mitarbeiterin am Centre for Sustainability Management und promoviert zum Thema Sustainable Supply Chain Management.

Stefan Schaltegger, Prof. Dr., ist Ordinarius für Betriebswirtschaftslehre, Leiter des Centre for Sustainability Management und des MBA Sustainability Management an der Leuphana Universität Lüneburg.

Anschrift: Centre for Sustainability Management (CSM), Leuphana Universität Lüneburg, Scharnhorststr. 1, 21335 Lüneburg, Tel.: +49 (0)4131/677-2260, E-Mails: erik.hansen@uni.leuphana.de; dharms@uni.leuphana.de; schaltegger@uni.leuphana.de

Die Autoren danken den anonymen Gutachtern für die wertvollen Hinweise zur Weiterentwicklung des Beitrags sowie dem deutschen Bundesministerium für Umwelt, Naturschutz und Reaktorsicherheit (BMU) für die Förderung der Studie, die die Grundlage für diesen Beitrag bildet. 